\title{
NUEVOS DATOS DE REFERENCIA PARA LA TASA DE CRECIMIENTO DE RHIZOCARPON SUBG. RHIZOCARPON EN PATAGONIA
}

\author{
KAREN BORRAZZO" \& IRENE A. GARIBOTTI*
}

\begin{abstract}
RESUMEN
Se presentan los resultados obtenidos en el relevamiento del tamaño de líquenes pertenecientes al género Rhizocarpon subgénero Rhizocarpon realizado en superficies expuestas durante la última centuria en ambientes áridos de la Patagonia (Santa Cruz, Argentina). Se relevó el cementerio de la ciudad de Puerto Deseado y superficies expuestas por el retroceso del Glaciar Túnel en el Parque Nacional los Glaciares, proveyendo ocho nuevos sitios de referencia para estimar la tasa de crecimiento de estos líquenes. La tasa de crecimiento para el subgénero Rhizocarpon en estos sitios se estima entre 0,125 y $0,061 \mathrm{~mm}$ por año, siendo concordante con los valores obtenidos en otros sectores de la estepa patagónica, y difiriendo significativamente de las tasas de crecimiento estimadas para regiones más húmedas de los Andes Patagónicos.
\end{abstract}

PALABRAS CLAVE: liquenometría, Rhizocarpon subg. Rhizocarpon, tasa de crecimiento, Patagonia.

\section{NEW DATA ON GROWTH RATE OF RHIZOCARPON SUBG. RHIZOCARPON LICHENS IN PATAGONIA}

\begin{abstract}
This paper presents the results from lichen size survey of genus Rhizocarpon subgenus Rhizocarpon performed on surfaces exposed during last century in arid Patagonian environments (Santa Cruz, Argentina). We surveyed the cemetery of Puerto Deseado City and rock surfaces exposed by the retreat of Túnel Glacier in Los Glaciares National Park, providing eight new reference sites to estimate the growth rate of these lichens. Growth rate estimations for subgenus Rhizocarpon are between 0,125 to 0,061 mm per year. These values agree with previous estimations for other portions of Patagonian steppe and exhibit significant difference with available estimations for wetter regions in the Patagonian Andes.
\end{abstract}

KEY WORDS: lichenometry, Rhizocarpon subg. Rhizocarpon, growth rate, Patagonia.

* CONICET-IMHICIHU, UBA. Saavedra 15 piso 5 (1083ACA), Buenos Aires. Email: kborrazzo@yahoo.com.ar.

* IANIGLA, CCT-CONICET, Mendoza. Av. Ruiz Leal s/n, Parque General San Martín, C.C. 330, Mendoza. Email: ireneg@ mendoza-conicet.gov.ar. 


\section{INTRODUCCIÓN}

En el año 2011 se inició un programa interdisciplinario entre el proyecto de arqueología de la Patagonia Meridional (Proyecto Magallania, dirigido por Luis Borrero) y el proyecto abocado a desarrollar técnicas liquenométricas para la reconstrucción de fluctuaciones glaciares en los Andes Patagónicos (dirigido por Irene Garibotti). Este programa interdisciplinario tiene el fin de explorar el uso de la liquenometría como herramienta en temas vinculados a la cronología y los procesos de formación del registro en la investigación arqueológica de Patagonia. Los estudios realizados proveyeron las primeras estimaciones para la tasa de crecimiento de Rhizocarpon subg. Rhizocarpon en la estepa patagónica y permitieron aplicar en forma exitosa métodos liquenométricos para estimar la edad mínima de construcción de estructuras arqueológicas (Borrazzo y Garibotti 2011; Borrero et al. 2011). Asimismo, estos estudios complementaron los realizados anteriormente en el área andina de la Patagonia (Garibotti y Villalba 2009), permitiendo hacer una discusión ecológica exhaustiva acerca de la variabilidad de las tasas de crecimiento del subgénero Rhizocarpon a lo largo del gradiente de precipitaciones oeste-este en la
Patagonia (Garibotti et al. 2011).

La liquenometría es un método de datación cuya exactitud depende fuertemente de la fidelidad con la que se han estimado las tasas de crecimiento del liquen bajo estudio (Benedict 2009). Por lo tanto, la incorporación de nuevos datos independientes, basados en superficies de referencia cuya edad puede acotarse con precisión, contribuye a aumentar la deteminación general del método. El objetivo del presente trabajo es proveer nuevos datos de referencia para estimar la tasa de crecimiento del subgénero Rhizocarpon en la estepa patagónica. Se informan los resultados obtenidos en el relevamiento del tamaño de líquenes de este subgénero en tumbas del cementerio de la ciudad de Puerto Deseado y en las superficies expuestas por el retroceso del Glaciar Túnel, ambos sitios en la Provincia de Santa Cruz (Fig. 1). Adicionalmente, se analiza esta información en relación a la disponible en estudios previos, y se discute las fortalezas y las limitaciones en la aplicación de métodos liquenométricos en la Patagonia.

\section{SITIOS DE ESTUDIO}

\section{Cementerio de Puerto Deseado}

La ciudad de Puerto Deseado, fundada en

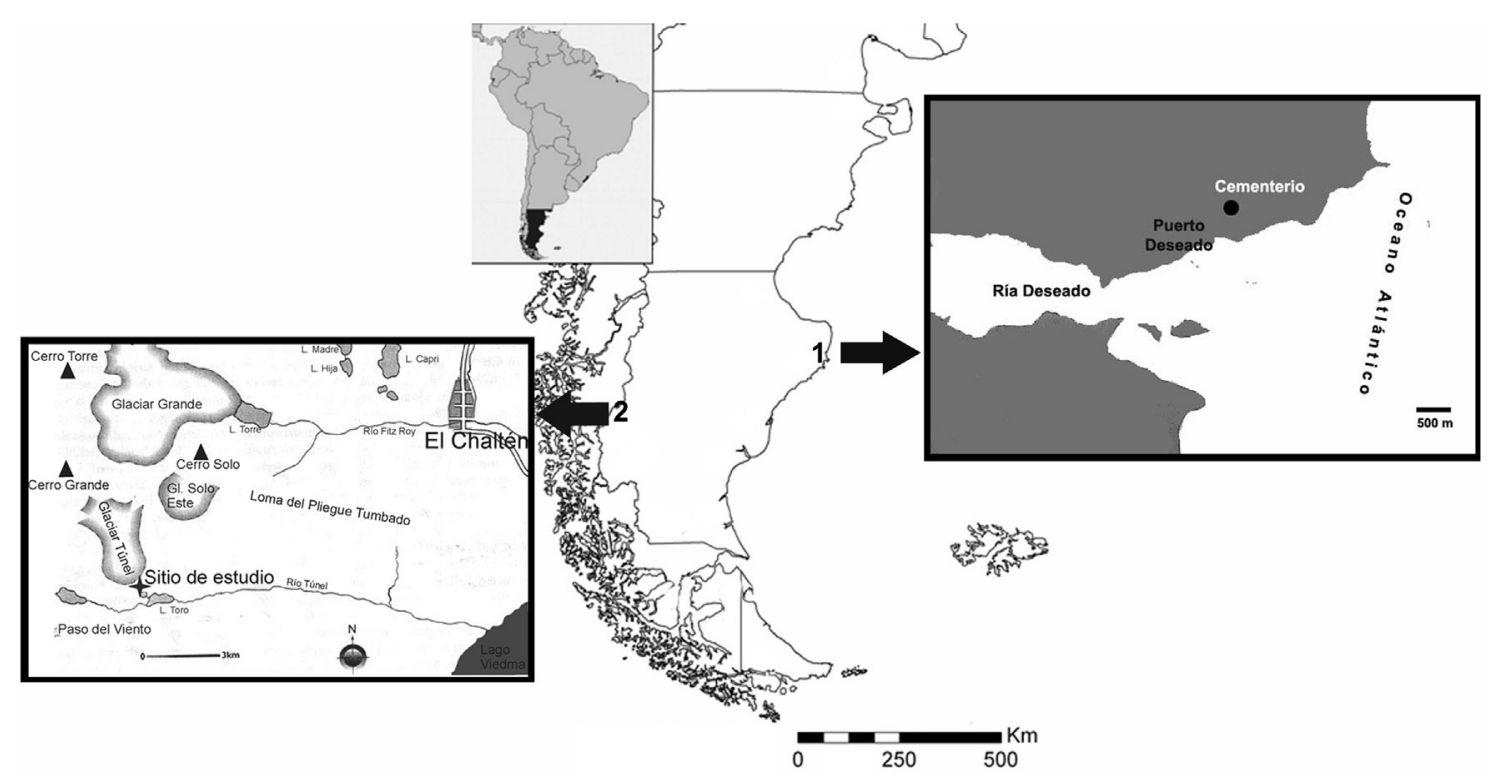

Fig. 1. Ubicación de los sitios de referencia para Rhizocarpon subg. Rhizocarpon informados en este trabajo: 1. Cementerio de Puerto Deseado. 2. Glaciar Túnel (Parque Nacional Los Glaciares). 
1883, se ubica sobre la margen norte de la ría del Deseado, a aproximadamente $2 \mathrm{~km}$ de su desembocadura. La temperatura media anual es de $9.8^{\circ} \mathrm{C}$ (media máxima estival: $28^{\circ} \mathrm{C}$; media mínima invernal: $-2^{\circ} \mathrm{C}$ ) y las precipitaciones medias anuales son de $202 \mathrm{~mm}$. El Cementerio Municipal (4744'46”S, 6552'24”O) se ubica hacia el noreste de la ciudad (Fig. 1). El mismo presenta una elevada frecuencia de lápidas de la primera mitad del siglo XX, realizadas sobre rocas de la formación Bahía Laura (ignimbritas de composición riolítica a riodacítica con coloraciones rojas a violáceas, Giacosa et al. 1998; Iantanos et al. 2009; Fig. 2). Estas materias primas, de disponibilidad local, fueron también utilizadas en la construcción edilicia de Puerto Deseado. En el cementerio se relevaron todas las tumbas, identificándose siete de ellas que presentaron colonización de individuos pertenecientes al subgénero Rhizocarpon. Los individuos identificados fueron medidos con regla flexible. Es importante destacar la elevada frecuencia de casos de lápidas colonizadas por Rhizocarpon subgénero Rhizocarpon registrados en este sitio, en relación al relativamente bajo número de lápidas con estos líquenes observado en cementerios de mayor envergadura, como los de Río Gallegos o Punta Arenas (Garibotti et al. 2011). Esto podría deberse al tipo de roca utilizada en la confección de las tumbas de Puerto Deseado, a diferencia de los otros cementerios en donde se utilizó principalmente cemento o mármol para su manufactura.

\section{Glaciar Túnel}

El Glaciar Túnel desciende desde el cerro Grande perteneciente al cordón Adela. El cordón Adela junto con el cerro Torre forman una cadena montañosa situada en el límite oeste del campo de hielo continental sur, cerca de los $49^{\circ} 20^{\prime}$ de latitud sur (Fig. 1). Toda la región está comprendida dentro del área norte del Parque Nacional los Glaciares. El clima en el área es templado

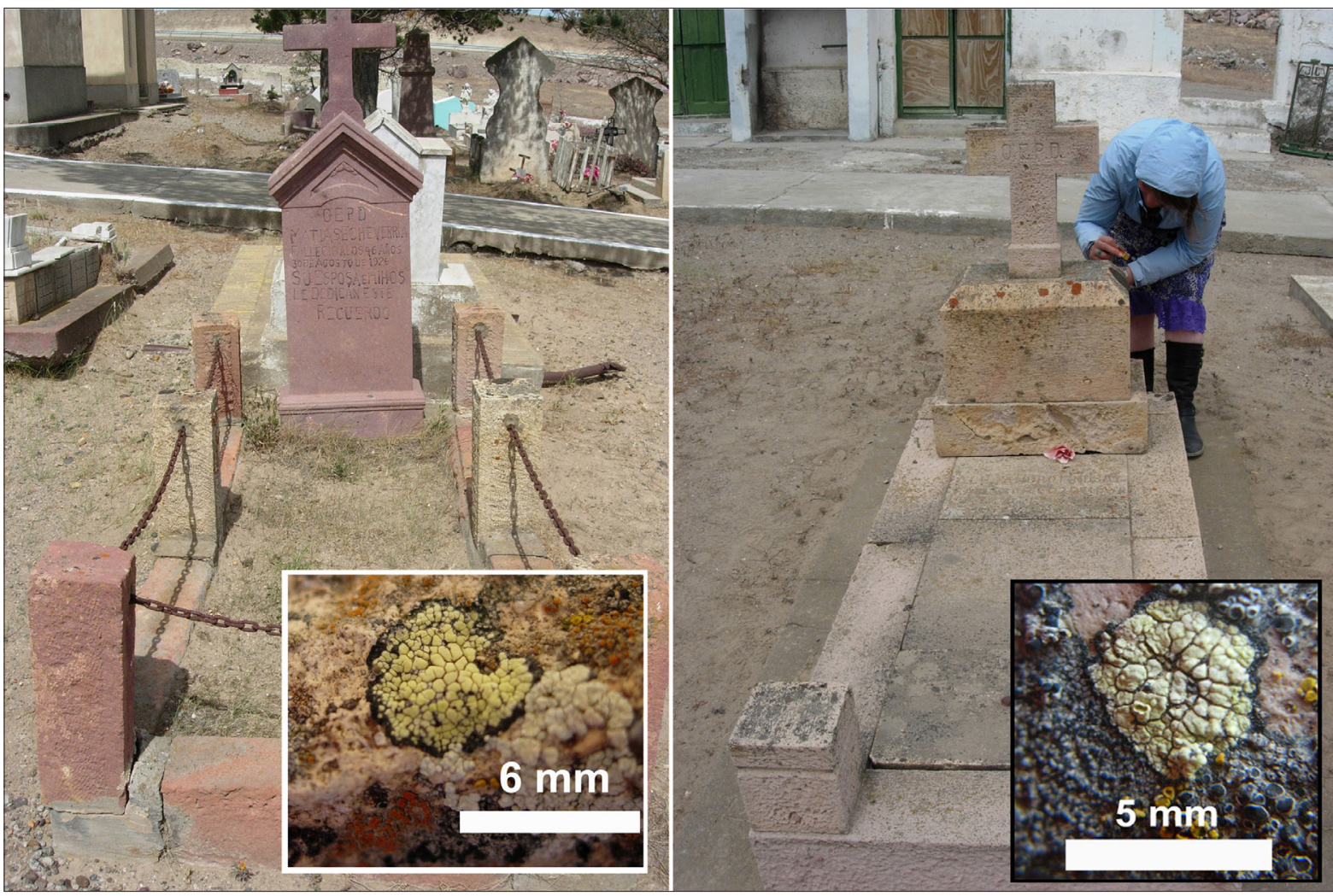

Fig. 2. Dos de las tumbas del cementerio de Puerto Deseado manufacturadas con rocas locales en las que se encontraron ejemplares del subgénero Rhizocarpon. Se incluye detalle de los líquenes medidos en cada caso. 
húmedo, con una temperatura media anual de $7,5^{\circ} \mathrm{C}$ (media invierno: $0,6^{\circ} \mathrm{C}$; media verano: $\left.13,4^{\circ} \mathrm{C}\right)$. Las precipitaciones están distribuidas casi homogéneamente durante el año, pero el área se caracteriza por importantes variaciones climáticas en la medida que nos alejamos de la cordillera. No existen registros de precipitaciones para el valle del Glaciar Túnel, pero la vegetación corresponde a una zona de transición entre la estepa patagónica y los bosques andinos, con mezcla de especies características del bosque y especies esteparias. Los fuertes vientos limitan el crecimiento vegetal en el área. El valle del Glaciar Túnel fue explorado tempranamente por expediciones de investigadores. En el año 1916 los alemanes Alfredo Kölliker, Lutz Witte y Franz Kühn realizaron una expedición recorriendo el valle del río Túnel, durante la cual realizaron un profuso registro fotográfico que fue publicado bajo el auspicio de la Sociedad Científica Alemana de Buenos Aires (Köllinker et al. 1917). Asimismo, Lliboutry (1952) realizó estudios glaciológicos en

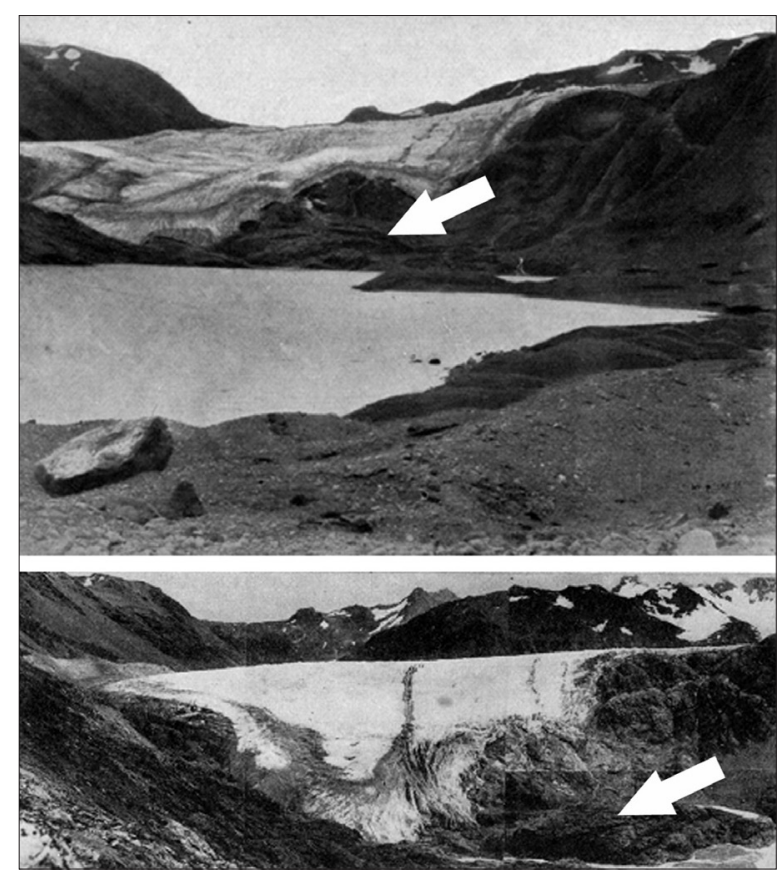

Fig. 3. Fotografías históricas del frente del Glaciar Túnel utilizadas para acotar la edad de exposición de los afloramientos rocosos en el frente del glaciar. Foto superior correspondiente al año 1916 (Köllinker et al. 1917), foto inferior correspondiente aproximadamente al año 1950 (Lliboutry 1952). Las flechas señalan el mismo rasgo topográfico en ambas fotografías. el área, dejando registro fotográfico del glaciar. Varias de estas fotos muestran la posición histórica del frente del Glaciar Túnel (Fig. 3), permitiendo diferenciar las zonas que ya se encontraban expuestas por el retroceso del glaciar en el momento de la expedición. En el presente trabajo se relevaron los afloramientos rocosos que en estas fotos históricas se observan frente al glaciar, considerando que dichas superficies habían sido recientemente expuestas por el retroceso de este último en el año 1916. Sobre estos afloramientos se midió el tamaño de líquenes del subgénero Rhizocarpon utilizando regla flexible.

\section{RESULTADOS Y DISCUSIÓN}

Los resultados de la medición de líquenes de subgénero Rhizocarpon en los sitios de referencia relevados se presentan en la Tabla 1. Los mismos son consistentes, mostrando un incremento en el tamaño de los talos en los sitios progresivamente más antiguos. Las tasas de crecimiento estimadas varían entre 0,061 y 0,125 mm por año, aumentando en función del tamaño del talo. Estos resultados son consistentes con los

Tabla 1. Datos liquenométricos de los sitios de referencia estudiados en la estepa patagónica. El diámetro de

Rhizocarpon subgénero Rhizocarpon se refiere al talo más grande encontrado en cada sitio.

\begin{tabular}{|c|c|c|c|c|c|}
\hline$\#$ & $\begin{array}{c}\text { Sitio de } \\
\text { referencia }\end{array}$ & $\begin{array}{c}\text { Fecha de } \\
\text { exposición }\end{array}$ & $\begin{array}{c}\text { Edad } \\
\text { estimada } \\
\text { del sitio }\end{array}$ & $\begin{array}{c}\text { Diámetro } \\
\text { (mm) }\end{array}$ & $\begin{array}{c}\text { Tasa de } \\
\text { crecimiento } \\
\text { (mm año) }\end{array}$ \\
\hline 1 & $\begin{array}{c}\text { Tumba } \\
\text { Alvarez }\end{array}$ & 1916 & 96 & 12 & 0,125 \\
\hline 2 & $\begin{array}{c}\text { Tumba } \\
\text { Aenelle }\end{array}$ & 1919 & 93 & 7 & 0,075 \\
\hline 3 & $\begin{array}{c}\text { Tumba } \\
\text { Echeverría }\end{array}$ & 1926 & 86 & 6 & 0,070 \\
\hline 4 & $\begin{array}{c}\text { Tumba } \\
\text { Longas }\end{array}$ & 1937 & 75 & 6 & 0,080 \\
\hline 5 & $\begin{array}{c}\text { Tumba } \\
\text { Sotelo }\end{array}$ & 1941 & 71 & 6 & 0,084 \\
\hline 6 & $\begin{array}{c}\text { Tumba } \\
\text { Lindsay }\end{array}$ & 1942 & 70 & 5 & 0,071 \\
\hline 7 & $\begin{array}{c}\text { Tumba } \\
\text { Cantella }\end{array}$ & 1946 & 66 & 4 & 0,061 \\
\hline 8 & $\begin{array}{c}\text { Glaciar } \\
\text { Túnel }\end{array}$ & 1916 & 97 & 12 & 0,124 \\
\hline
\end{tabular}


publicados anteriormente para otras regiones de la estepa Patagónica, habiéndose discutido que el aumento de la tasa de crecimiento con la edad puede corresponderse con una fase madurativa del ciclo de vida de los líquenes que se caracteriza por crecimiento acelerado (Garibotti et al. 2011).

Los nuevos sitios de referencia $(n=8)$ presentados en este trabajo constituyen un incremento significativo con respecto a los disponibles previamente $(n=6)$ para la estepa Patagónica, permitiéndonos así mejorar la confianza en las estimaciones de la tasa de crecimiento del subgénero Rhizocarpon, y por ende la precisión en los resultados de las dataciones realizadas utilizando métodos liquenométricos. Las lápidas de cementerios representan sitios de referencia cuya edad se puede acotar con gran precisión. Aún cuando es posible que la lápida haya sido construida algunos años posteriores a la fecha de deceso de la persona, se puede esperar que este error sea muy pequeño y por ello tenga un efecto limitado en las estimaciones de las tasas de crecimiento. Asimismo, la edad del sitio de referencia al frente del Glaciar Túnel pudo acotarse con precisión mediante el uso de fotografías históricas con fechas conocidas. Por lo tanto, puede plantearse que los sitios de referencia disponibles para la estepa patagónica son de exactitud y que las tasas de crecimiento pueden usarse confidentemente para estimar la edad mínima de exposición de una superficie.

Las estimaciones disponibles para la tasa de crecimiento del subgénero Rhizocarpon se extienden durante los últimos 120 años (este trabajo y Garibotti et al. 2011). Estrictamente no es posible extrapolar las tasas de crecimiento a otras edades, debido a que ellas varían con la edad del liquen. Por lo tanto, esta representa actualmente la mayor limitación en la aplicación de técnicas liquenométricas en la estepa Patagónica, siendo necesario intensificar los esfuerzos para identificar estructuras arqueológica o geológicas de mayor antigüedad que puedan ser utilizadas como sitios de referencia.

\section{AGRADECIMIENTOS}

Esta investigación ha contado con el apoyo económico de CONICET, la ANPCyT (PICT2317), y el Instituto Interamericano para Investigación en Cambio climático (IAI, CRN II \# 2047).

\section{BIBLIOGRAFÍA}

Benedict, J. B. (2009). A review of lichenometric dating and its applications to archaeology. American Antiquity, $74,143-172$

Borrazzo, K., \& Garibotti, I.A (2011). Liquenometría y Arqueología en Fuego-Patagonia. Libro de resúmenes de las VIII Jornadas de Arqueología de la Patagonia (p. 18), Malargüe, Mendoza.

Borrero, L.A., Borrazzo, K., Garibotti, I.A., \& Pallo, M.C. (2011). Concentraciones de pilas de roca en la cuenca superior del río Santa Cruz (Argentina). Magallania, 39(2), 193-206.

Garibotti, I.A, Borrazzo, K., \& Borrero, L.A. (2011). Aplicación de técnicas liquenométricas en la arqueología de Patagonia utilizando Rhizocarpon subgénero Rhizocarpon. En L.A. Borrero \& K. Borrazzo (Comps.) Bosques, Montañas y cazadores: investigaciones arqueológicas en Patagonia Meridional (pp. 85-103). Buenos Aires: CONICET-IMHICIHU.

Garibotti, I.A., \& Villalba, R. (2009). Lichenometric dating using Rhizocarpon subgenus Rhizocarpon in the Patagonian Andes, Argentina. Quaternary Research, 71, 271-283

Giacosa, R. E., Césari, O., \& Genini, A. (1998). Descripción de la Hoja Geológica 4766 - III y IV. Puerto Deseado, Provincia de Santa Cruz, Tomo 240 de Boletín del Programa Nacional de Cartas Geológicas de la Republica Argentina 1:250.000. Buenos Aires: Ministerio de Economía.

Iantanos, N., Moreno, J.E., Andolfo, M.A., Isla, F., \& Castro, A. (2009). Características y evolución del tómbolo Cabo Blanco, Provincia de Santa Cruz, Argentina. Naturalia Patagónica, 4(2), 33-45.

Kölliker, A., Kühn, F., Reichert, A., Tomsen, A., \& Witte, L. (1917). Patagonia: Resultados de las expediciones realizadas de 1910 a 1916. Buenos Aires: Sociedad Cientifica Alemana.

Lliboutry, L. (1952). Estudio cartográfico, geológico y glaciológico de la zona del Fitz Roy. Serie Ensayos 17. Buenos Aires: Instituto de Geografía, Facultad de Filosofía y Letras, Universidad de Buenos Aires. 
